\title{
Социологический анализ распространения виртуальных мобильностей как формы виртуализации пространственных перемещений (на основании социологии мобильностей Джона Урри)
}

\author{
Ходыкин А.В. \\ Самарский государственный экономический университет, \\ Россия, 443090, г. Самара, ул. Советской армии, 141 \\ E-mail: khodykin8@gmail.com
}

\begin{abstract}
Аннотация. Представлено исследование виртуальных мобильностей как возможностей преодоления сопротивления физического пространства посредством создания его виртуального образа. На основании трудов британского социолога Джона Урри автором исследованы виртуальные мобильности с точки зрения социологии пространства. Анализ социологии мобильностей Урри позволил выделить в его работах три хронологических этапа становления мобильностей, связанных с изобретением и внедрением новой техники и технологий. Первый этап (XIX век) связан с механизацией передвижения. Второй этап (XX век) включает в себя массовое производство двигателей внутреннего сгорания, их совершенствование и появление новых видов транспорта. Третий этап (рубеж XX-XXI веков) характеризует революционное развитие коммуникации за счёт резкого повышения мобильности информации, что сделало возможным активное развитие виртуальных мобильностей. Виртуальные мобильности делают отдалённые в физическом пространстве события близкими в виртуальном пространстве и доступными для виртуального участия в них. Автором исследованы сервисы онлайн карт, содержащие панорамные изображения; спутниковые снимки и фотографии населённых пунктов; видеозаписи поездок; фильмы о путешествиях; виртуальные экскурсии; образовательные виртуальные мобильности; видеопутешествия по экстремальным местам и виртуальные путешествия в космическом пространстве. Социология мобильностей Урри стала исходной точкой для теоретизирования, формирующего социологию виртуальных мобильностей. Все остальные концепции, будь то социология пространства или теории виртуальности, становятся дополнительными ресурсами для теоретизирования.
\end{abstract}

Ключевые слова: Джон Урри, виртуальная реальность, пространство, социальная теория.

Для цитирования: Ходыкин А.В. 2020. Социологический анализ распространения виртуальных мобильностей как формы виртуализации пространственных перемещений (на основании социологии мобильностей Джона Урри). NOMOTHETIKA: Философия. Социология. Право. 45 (3): 579-588. DOI 10.18413/2712-746X-2020-44-3-579-588

\section{Sociological analysis of the spread of virtual mobility as a form of spatial movement virtualization (based on John Urry's sociology of mobility)}

\author{
Alexander V. Khodykin \\ Samara State Economic University, \\ 141 Sovetskoy Armii St, Samara, 443090, Russia \\ E-mail: khodykin8@gmail.com
}

Abstract. The paper presents a study of virtual mobility as a way to overcome the resistance of physical space by creating a virtual image of it, which allows a person to be in a different place from where he is physically, 
through virtual reality. Based on the works of the British sociologist John Urry, the author investigated virtual mobility from the point of view of the sociology of space. The analysis of the sociology of mobility of Urry allowed us to identify three chronological stages of the formation of mobility associated with the invention and introduction of new techniques and technologies. The first stage (XIX century) is associated with the mechanization of movement. The second stage (XX century) includes mass production of internal combustion engines, their improvement and the emergence of new modes of transport. The third stage (the turn of the XXXXI centuries) characterizes the revolutionary development of communication due to a sharp increase in the mobility of information, which made possible the active development of virtual mobility. Virtual mobility makes events that are remote in physical space close in virtual space and available for virtual participation in them. The author has studied the following information technology capabilities: online map services containing panoramic images; satellite images and photos of localities; video recordings of trips; travel films; virtual excursions; educational virtual mobility; video trips to extreme places and virtual travel in Outer Space. Urry's sociology of mobility has become the starting point for the theorizing that forms the sociology of virtual mobility. All other concepts, whether it is the sociology of space or the theory of virtuality, become additional resources for theorizing.

Keywords: John Urry, mobility, virtual reality, space, social theory.

For citation: Khodykin A.V. 2020. Sociological analysis of the spread of virtual mobility as a form of spatial movement virtualization (based on John Urry's sociology of mobility). NOMOTHETIKA: Philosophy. Sociology. Law series. 45 (3): 579-588 (in Russian). DOI 10.18413/2712-746X-2020-44-3-579-588

\section{Введение}

Пожалуй, наибольший вклад в теорию пространственных мобильностей за всю историю социологической мысли внёс британский социолог Джон Урри, создавший оригинальную теорию мобильностей, исследующую перемещение людей не в социальном, как это делали большинство социологов, начиная с Питирима Сорокина [Сорокин, 1992], а в физическом пространстве. В своей работе «Мобильности» Джон Урри доказывает на множестве исторических примеров и технических фактов тезис о том, что развитие технологий даёт людям новые возможности для пространственных перемещений, которые становятся основой жизни и развития современной цивилизации [Урри, 2012]. «Движение как ключевой социологический феномен и понимание организации всей социальной жизни через конкретно-исторические исследования социальных и технических систем, обеспечивающих это движение», - именно так Н.А. Харламов обозначил основное кредо социологии мобильностей Урри [Харламов, 2012, с. 10].

Пространственное устроение социальной жизни, а также влияние особенностей физического пространства на действия социальных акторов исследуются социологией пространства [Lefebvre, 1974; Филиппов, 2001; 2003; 2008; Звоновский, 2009; Ходыкин, 2019a]. Физическое пространство оказывает сопротивление действиям социальных акторов посредством дистанций, которые акторам необходимо преодолевать, и свойств среды, в которой локализованы действия акторов. Чтобы переместиться из точки А в точку Б, нужно затратить усилия, объём которых зависит от дистанции между точками в пространстве и особенностей среды, в которой происходит перемещение. Таким образом, мобильности в физическом пространстве есть результат преодоления акторами сопротивления данного пространства. Совершенствование технологий повышает эффективность преодоления человеком сопротивления физического пространства, в результате чего происходит то развитие мобильностей, которое стало центральной темой социологии Джона Урри. Прочтение теории мобильностей Урри в контексте социологии пространства даёт возможность проследить то, как увеличение возможностей человека преодолевать сопротивление физического пространства приводит к совершенствованию уже имеющихся и появлению новых видов мобильностей, а также выделить виртуальные мобильности как осо- 
бый вид преодоления сопротивления физического пространства, характерный для информационной эпохи. Решению этих задач посвящена данная работа.

\section{Анализ теории мобильностей Урри в контексте социологии пространства}

На основании анализа творчества Джона Урри можно выделить три ключевых этапа формирования мобильностей, связанных с изобретением и внедрением парадигмально новой техники и технологий, революционно изменивших способность человека к эффективному преодолению сопротивления физического пространства.

Первый этап (XIX век). Механизация передвижения. До этого времени передвижение осуществлялось за счёт тяговой силы животных на суше и ветра на воде (паруса). Появление двигателей резко повысило скорость и грузоподъёмность средств передвижения, что нашло наиболее значимое отражение в железнодорожном транспорте. Появление железных дорог дало людям возможность быстро перемещаться на бо́льшие расстояния, что в прежние времена было невозможным. Это привело к бурному развитию туризма в конце XIX века, когда путешествия стали доступны относительно широкому кругу населения развитых стран. Развитию туризма как особого вида массовых мобильностей Урри уделяет отдельное внимание в работах, посвящённых исследованию феномена «взгляда туриста» [Urry, 1990; 2000].

Второй этап (XX век). Массовое производство двигателей внутреннего сгорания, их совершенствование и появление новых видов транспорта. Конвейерное производство двигателей сделало доступными личные автомобили, что повысило мобильность большего числа людей. Совершенствование двигателестроения сделало возможным создавать крупные суда с мощными двигателями, в результате чего мобильность грузов значительно повысилась за счёт резкого повышения грузоподъёмности, безопасности и скорости передвижения по морю. Появление авиации дало человеку возможность перемещаться в новом воздушном пространстве, специфика которого позволяет преодолевать его со значительно большей скоростью. Появление авиации и активное использование автомобилей сделали возможным массовое перемещение людей, товаров и технологий по всей Земле. В работах, посвящённых «автомобильности», Урри характеризует развитие автомобилестроения и повышение доступности автомобилей как важнейший драйвер развития глобальных мобильностей как товаров, так и людей [Urry, 1999; 2003; 2004; Featherstone et al., 2005]. Для перемещения товаров революционное значение, по Урри, имеет появление контейнерных морских перевозок, значительно удешевивших транспортировку грузов. Современные суда, гружённые огромным количеством контейнеров, заполненных товарами, способны относительно быстро и с меньшей по сравнению с другими видами перевозок стоимостью перевозить грузы в отдалённые от их производства точки Земли. Книгу «Офшоры» Урри посвятил формированию новых экономических отношений под влиянием развития мобильностей. Автор показывает, как широкие возможности перемещения рабочей силы, товаров и производств способствуют созданию офшорных зон, экспорту производства и его издержек из развитых в менее развитые страны, формированию новых видов социально-экономического неравенства, как между странами, так и между отдельными людьми и появлению других социальных рисков [Урри, 2017].

Третий этап (рубеж XX-XXI веков). Революционное развитие коммуникации за счёт резкого повышения мобильности информации. Сегодня наиболее эффективно преодолевать сопротивление физического пространства способны информационные потоки, мобильность которых перестала зависеть от мобильности их материальных носителей. Развитие информационных технологий позволило людям даже на дальних расстояниях мгновенно обмениваться информацией и тиражировать её, что привело к четвёртой промышленной революции и появлению информационного общества. Для этого этапа харак- 
терно развитие нового вида мобильностей, связанного с телекоммуникационными технологиями и появлением Интернета - виртуальных мобильностей, о которых мы подробно поговорим далее.

Джон Урри, разрабатывая социологию мобильностей, выделяет в ней также и системы мобильностей, которые определяются как «комплекс социальных отношений и материальной инфраструктуры, который делает определенный вид перемещения возможным» [Харламов, 2012, с. 24]. Системы мобильностей создают социальную и предметную структуру, упорядочивающую свободные потоки мобильностей. Если мобильности представляют собой способ преодоления сопротивления физического пространства для перехода в иную его точку, то системы мобильностей - это инструменты, обеспечивающие наиболее эффективный переход в иную точку физического пространства. В контексте виртуальных мобильностей системы мобильностей создаются системами материальных объектов, делающих виртуальную реальность возможной, т.е. видеокамер, серверов, компьютеров и т.д., а также правил и законов, регулирующих виртуальные мобильности.

Джон Урри классифицирует мобильности на пять видов [Урри, 2012]:

1. Физическое перемещение людей в пространстве. Подробно охарактеризовано выше при описании первых двух этапов формирования мобильностей. Сопротивление физического пространства преодолевается здесь телами людей.

2. Физическое перемещение объектов в пространстве. Также охарактеризовано выше при описании первых двух этапов формирования мобильностей. Сопротивление физического пространства преодолевается различными техническими объектами и грузами.

3. Воображаемые путешествия. Это «путешествия», осуществляемые в сознании человека при помощи имеющихся у него образов пространств, полученных из книг, фильмов, рассказов, фотографий, картин и т.д. Такие путешествия стали возможны благодаря совершенствованию знаний человечества, повышению их массовой доступности и информированности людей относительно пространственно отдалённых регионов в рамках становления информационного общества. Сопротивление физического пространства в данном случае преодолевается не при помощи пространственного перемещения людей и объектов, а силой воображения и ресурсами накопленных человечеством знаний. В таком случае недостаток ресурсов, необходимых для физических путешествий, компенсируется информационными ресурсами.

4. Коммуникационные мобильности, осуществляемые «посредством обмена $\mathrm{CMC}$, текстами, письмами и телеграммами, через факс, телефон или мобильный телефон» [Урри, 2012, с. 136]. Сопротивление физического пространства преодолевается информационными потоками и коммуникационными сетями, делающими пространственно отдалённых социальных акторов коммуникационно близкими.

5. Виртуальные мобильности - это возможность быть не в то время или не в том месте, в котором человек находится физически, и видеть, слышать, чувствовать что-либо, пребывать в какой-либо ситуации посредством виртуальной реальности. Виртуальное пространство становится цифровым образом реального физического пространства [Хоружий, 1997; Грицанов и др., 2003; Рузавин, 2010]. Преодоление сопротивления физического пространства осуществляется за счёт его замещения виртуальным аналогом, путешествовать в котором значительно проще. Виртуальные мобильности делают отдалённые в физическом пространстве и (или) во времени события близкими и доступными для пусть и виртуального, но всё же участия в них. Телевизионные трансляции, виртуальные экскурсии по музеям, видеосъёмки природных объектов, видеозаписи лекций - всё это примеры виртуальных мобильностей. Виртуальные мобильности можно разделить на трансляционные и нетрансляционные. Первые предполагают удалённое виртуальное участие в событии в одно время с его совершением. К ним относятся прямые трансляции, изображения с камер наблюдения в онлайн режиме, вебинары и т.п. Для вторых не выполняется требование единства во времени события и виртуального участия в нём актора. Сюда можно от- 
нести видео- и аудиозаписи лекций, видеоэкскурсии по музеям, телепередачи о путешествиях в дальние страны и т.д.

Анализ корпуса работ Джона Урри в контексте социологии пространства показывает, что социолог акцентировал внимание на создании научно-техническим прогрессом новых средств передвижения и более совершенной инфраструктуры (систем мобильностей) как ресурсов для трансформирующего социальные явления более эффективного преодоления сопротивления физического пространства. В своей работе «Мобильности» он также выделяет, но не исследует подробно воображаемые путешествия, коммуникационные и виртуальные мобильности [Урри, 2012]. Однако, не интегрируя ресурсы социологии пространства в свою теорию мобильностей, Урри не уделил достаточного внимания исследованию создаваемой модели физического пространства в виртуальном пространстве, не оказывающем физического сопротивления перемещениям в нём. Таким образом, более эффективно преодолевать сопротивление физического пространства человек может посредством (1) совершенствования средств передвижения и необходимой для их использования инфраструктуры, (2) создания электронных коммуникационных сетей для мобильностей потоков информации, (3) создания в виртуальном пространстве моделей физического пространства и событий, объектов и перемещений в нём. В первом случае осуществляется мобильность физических объектов (человека и техники) в физическом пространстве, во втором - мобильность информации в физическом пространстве, а в третьем - мобильность воспринимаемых человеком виртуальных образов реальных перемещений, событий или объектов в виртуальном пространстве. Подробнее исследуем виртуальные мобильности на комплексе примеров.

\section{Исследование новых возможностей для виртуальных мобильностей}

На сегодняшний день, благодаря развитию цифровых технологий и их массовому внедрению в жизнь людей появляется большое количество новых возможностей для виртуальных мобильностей. Проанализируем некоторые недавно появившиеся возможности.

1. Сервисы онлайн-карт, предоставляющие возможности использования панорамных изображений, снимков со спутников и размещённых пользователями фотографий. Сегодня большинство сервисов онлайн-карт содержат функцию просмотра панорамных изображений и фотографий, покрывающих большинство населённых пунктов Земли. Панорамные изображения, созданные на основании данных, полученных путешествующими по населённым пунктам автомобилями с панорамными камерами, позволяют человеку не только осмотреть местность съёмки с возможностью вращения камеры на 360 градусов, но и возможность передвигаться по карте при помощи специальных стрелок. В результате пользователь получает ощущение полного присутствия на дорогах и в населённых пунктах. Причём в некоторых случаях есть возможность сравнить состояние данной местности с её состоянием в предыдущие годы при помощи выбора года съёмки. В таком случае виртуальная мобильность становится возможной не только в пространстве, но и во времени. Спутниковая карта Земли даёт возможность просматривать сверху практически всю поверхность нашей планеты. А возможность размещения фотографий на электронных картах предполагает коммуникацию между пользователями, добавляющими на местность её фотографии. Люди активно добавляют свои фотографии деревень, природных объектов и городских пейзажей, конкретизируя тем самым карту местности изображениями мест. Особого внимания заслуживает специально созданный для виртуальных путешествий проект Google Earth, создающий 3-D карту Земли. Такая карта представляет собой виртуальную модель Земли, предназначенную для обзора и перемещения по ней, т.е. для мобильности пользовательского восприятия в виртуальной модели физического пространства Земли - 3-D карте. При этом возможности виртуальных мобильностей уже выходят 
за пределы Земли. Корпорация Google совместно с партнёрами создала и активно развивает проект создания 3-D карты Марса ${ }^{1}$. Таким образом, виртуально путешествовать, хотя пока и со значительными ограничениями, можно уже и по Марсу.

2. Дорожные видеозаписи. В последнее время на видеохостингах стали активно размещаться видеозаписи поездок, в особенности, железнодорожных. Появляются тематические каналы, полностью посвящённые таким видео. Причём никаких рассказов в них нет: просто размещается камера у окна, пишется звук, а зритель получает аудиовизуальное ощущение поездки ${ }^{2}$, симулирующее реальное железнодорожное путешествие. Такие видеозаписи содержат поездки в разное время суток и года по разным ландшафтам местности и характеризуются большой длительностью видео - встречаются записи, длящиеся по 12-15 часов. Примечательно, что они имеют большую популярность у пользователей: видео железнодорожных поездок набирают более сотни тысяч просмотров, что свидетельствует о наличии у людей спроса на такие виртуальные поездки. В наиболее популярных комментариях к таким видео пользователи отмечают создающееся у них ощущение реальности поездки и делятся воспоминаниями о своих реальных путешествиях, а также отмечают ценность для них возможности совершать железнодорожные путешествия, не покидая дома. Виртуальная мобильность в данном случае создаётся восприятием реальной мобильности в физическом пространстве (поездки), отражённой в его виртуальной модели (видеозаписи этой поездки).

3. Фильмы о путешествиях. Такие фильмы, рассказывающие о путешествиях в интересные точки нашей планеты, ранее снимались телевизионными компаниями, но в последнее десятилетие стали сниматься и видеоблогерами. В том числе благодаря подобным фильмам пространство присутствия отдельно взятого человека значительно расширяется, так как виртуально человек может посетить гораздо больше мест, чем реально. Однако в данном случае есть важное ограничение: человеку представляется возможность увидеть мир глазами авторов фильма, т.е. чужими глазами, в результате чего внимание зрителей акцентируется по желанию авторов фильмов. Здесь виртуальная мобильность создаётся посредством восприятия зрителем передаваемого автором нарратива, отражающего результат реальной мобильности автора в физическом пространстве (путешествия).

4. Виртуальные экскурсии. Уже в 2000-е годы люди смогли увидеть экспонаты Эрмитажа, картины Третьяковской галереи и другие культурные реликвии, не выходя из дома. Это стало доступным благодаря появлению виртуальных экскурсий по главным музеям и галереям разных стран. Люди получили возможность не только во всех подробностях и со всеми визуальными эффектами увидеть достопримечательности, но и послушать увлекательное повествование о них от специалистов в области истории, культурологии, искусствоведения и т.д. В данном случае виртуальная мобильность осуществляется в виртуальном пространстве, сконструированном авторами посредством сочетания виртуальных объектов, не имеющих реального воплощения, нарисованных с помощью компьютерных технологий и создающих виртуальную гиперреальность, и виртуальных моделей реально существующих или существовавших объектов (снимков произведений искусства, видеозаписей выступлений лектора и т.д.).

${ }^{1}$ Cм. URL: http://mars3dmap.com/\#bookmark. Чтобы виртуально путешествовать по Марсу в лучшем качестве и с большими возможностями, нужно скачать приложение от Google по URL: https://www.google.com/intl/ru/earth/download/gep/agree.html и там в меню необходимо выбрать пункт - «планета Марс». Инструкции по выполнению всех необходимых действий см. URL: https://www.google.com/earth/ (дата обращения: 09.03.2020).

${ }^{2}$ Примеры таких видео: URL: https://www.youtube.com/watch?v=6t5yTlm_3s8, https://www.youtube.com/watch?v=DyYszhSST04, https://www.youtube.com/watch?v= GFQnOL-_ aoI (дата обращения: 09.03.2020). 
5. Образовательные виртуальные мобильности. Информационные технологии позволяют создавать виртуальное образовательное пространство, объединяющее образовательные информационные ресурсы из разных стран, городов, университетов. Видеолекции, онлайн курсы, вебинары и другие информационные ресурсы позволяют людям из любой точки планеты, где есть Интернет, виртуально посещать научные и образовательные мероприятия лучших учебных заведений мира, находящихся в разных странах. Виртуальная мобильность здесь может осуществляться посредством мобильности восприятия слушателя (1) в виртуальных моделях реальных событий (лекций) или (2) в виртуальном пространстве, дополненном элементами гиперреальности, в котором реальное событие (лекция) дополняется гиперреальными эффектами (компьютерной графикой, иллюстрирующей слова лектора).

6. Видеосъёмки наиболее экстремальных мест. Современная техника обладает высоким запасом прочности и способна записать видео и звук даже из самых экстремальных мест. Поэтому сегодня мы можем виртуально заглянуть в жерло вулкана, в Марианскую впадину, на льды Северного и Южного полюсов и другие подобные места. По Урри, данная техника становится продолжением взгляда человека в другое пространство, протезирующим объектом, дополняющим и компенсирующим несовершенство человеческого тела, которое не способно пребывать в экстремальных средах. Поэтому виртуальная мобильность становится единственным способом преодоления восприятием человека сопротивления физического пространства самых экстремальных мест.

7. Виртуальные путешествия по космосу. Фото- и видеокадры с МКС, планет и спутников Солнечной системы, а также фильмы о космосе, показывающие сконструированные изображения даже далёких от нас галактик, позволяют человеку увидеть то, что он в принципе не может увидеть своими глазами, побывать, хотя и виртуально, там, где реально побывать человеку невозможно. Стоит также отметить, что применительно к космической деятельности виртуальные мобильности используются не только на Земле. Примером виртуальных мобильностей может стать и психологическая поддержка космонавтов на МКС посредством аудиовизуальных образов, при которой космонавтам, находящимся в длительных экспедициях вдали от родной планеты, показывают фотографии и видео с Земли, содержащие изображения природы, родных членам экипажа мест на Земле, близких им людей и т.д. В данном случае космонавты посредством таких виртуальных мобильностей в своём сознании преодолевают сопротивление пространства, породившее их отдалённость от родного для них мира [Ходыкин, 2019б].

Список охарактеризованных нами здесь виртуальных мобильностей отнюдь не исчерпывает всю их палитру. Но представленные выше их виды остаются наиболее распространёнными и демонстрируют основные способы формирования виртуальных мобильностей. Мы надеемся, что даже небольшой комплекс рассмотренных здесь мобильностей хотя бы частично способен продемонстрировать многообразие виртуальных путешествий. Создание виртуальных образов реальных объектов делает эти объекты более близкими нам и удобными для нашего восприятия. В результате пространство присутствия (пусть даже и виртуального) человека в мире значительно расширяется и включает в себя пространства новых объектов, начиная от жерл вулканов и заканчивая космическими объектами.

Даже в современном мире, где у человека появились более широкие возможности для физических мобильностей, на него всё ещё действуют значительные пространственные ограничения, препятствующие его физическим перемещениям. Однако создание виртуальной модели реального физического пространства предлагает новый способ преодоления его сопротивления и значительно расширяет пространство пребывания человека. Количество мест, в которых среднестатистический современный человек побывал при помощи виртуальных путешествий, сильно превышает количество физически посещённых им территорий. Высокая популярность использования охарактеризованных нами видов виртуальных путешествий свидетельствует о них как о значимом социальном явле- 
нии, изучение которого представляет высокий интерес для современной социологии. Поэтому виртуальные мобильности, которые активно развиваются в настоящее время, становятся предметом социологического анализа, теоретический инструментарий для которого был оставлен нам в наследство выдающимся британским социологом Джоном Урри.

\section{Заключение}

Преимущество социологии мобильностей Урри для исследования виртуальных мобильностей состоит в том, что Урри сумел создать теоретическую модель, позволяющую объединить перемещения в пространстве как источник производства социального в современном мире и виртуальную реальность как модель реального пространства в виртуальном. Использование теоретических ресурсов социологии пространства в контексте социологии мобильностей Урри позволило нам определить мобильности как способ преодоления сопротивления физического пространства. В случае мобильностей реальных объектов и людей в физическом пространстве его сопротивление преодолевается за счёт развития транспортных средств (социотехнических гибридов) и создания инфраструктуры (системы мобильностей). В случае виртуальных мобильностей это сопротивление преодолевается путём создания в виртуальном пространстве модели реального пространства, не оказывающего сопротивления перемещениям в нём. Таким образом, можно говорить о социологии виртуальных мобильностей, изучающей их как новый вид преодоления сопротивления физического пространства, ставший возможным благодаря развитию информационных технологий и появлению виртуальной реальности. Социология виртуальных мобильностей формируется на основании применения ресурсов социологии пространства к изучению виртуальных мобильностей в контексте социологии мобильностей Джона Урри.

Следующим теоретическим ходом становится применение ресурсов современной философии [Бодрийяр, 2017; Хоружий, 1997] и социологии [Иванов, 2002] виртуальности к социологии виртуальных мобильностей, что позволяет разделить виртуальное пространство на 1) виртуальный образ реального пространства и объектов в нём (например, панорамные изображения в онлайн-картах или видеозаписи поездок) и 2) гиперреальность, не имеющую воплощения в реальном пространстве (например, местность в компьютерной игре, нарисованная с помощью компьютерной графики). Это разделение отмечено нами при исследовании видеолекций, в которых видеозапись становится виртуальном образом реального события в реальном пространстве, а нарисованные с помощью компьютерной графики элементы (виртуальная электронная доска, например) относятся к гиперреальности и становятся её элементами в виртуальном пространстве как виртуальном образе реального пространства. В контексте социологии виртуальных мобильностей виртуальное пространство понимается только в первом его значении, т.е. как виртуальный образ реального пространства, пусть даже и дополненный элементами гиперреальности. Исключение из анализа гиперреальности обусловлено тем, что социология виртуальных мобильностей рассматривает виртуальное пространство не как самоценную реальность, а как особое средство преодоления сопротивления физического пространства с наибольшей результативностью при наименьших затратах.

Значимость социологии мобильностей Урри состоит в том, что она стала основой и исходной точкой для теоретизирования, формирующего социологию виртуальных мобильностей. Все остальные концепции, будь то социология пространства или теории виртуальности, становятся дополнительными ресурсами для теоретизирования.

\section{Список литературы}

1. Бодрийяр Ж. 2017. Симулякры и симуляция. Пер. с франц. М., Постум, 320 с.

2. Грицанов А.А., Галкин Д.В., Карпенко И.Д. 2003. Виртуальная реальность. Новейший философский словарь. Сост. и гл. науч. ред. А.А. Грицанов. 3-е изд., исправл. М., Книжный Дом: 171-173. 
3. Звоновский В.Б. 2009. Социология пространства повседневности. Самара, Самарский университет, $166 \mathrm{c.}$

4. Иванов Д.В. 2002. Виртуализация общества. Версия 2.0. СПб., Петербургское Востоковедение, $217 \mathrm{c}$.

5. Рузавин Г.И. 2010. Виртуальность. Новая философская энциклопедия. Электронная книга. URL: https://iphlib.ru/library/collection/newphilenc/document/HASH01b7eff9048db8416a128fb7 (дата обращения: 18 апреля 2020).

6. Сорокин П.А. 1992. Человек. Цивилизация. Общество. Пер. с англ. М., Политиздат, 543 с.

7. Урри Дж. 2012. Мобильности. Пер. с англ. М., Праксис, 501 с.

8. Урри Дж. 2017. Офшоры. Пер. с англ. М., Дело, РАНХиГС, 288 с.

9. Филиппов А.Ф. 2008. Социология пространства. СПб., Владимир Даль, 285 с.

10. Филиппов А.Ф. 2001. Социология пространства как теоретическая альтернатива. В кн.: Россия: трансформирующееся общество. М., КАНОН-пресс-Ц, 45-63.

11. Филиппов А.Ф. 2003. Теоретические основания социологии пространства. Автореф. дис. ... докт. соц. наук. М., 40 с.

12. Харламов Н.А. 2012. Новое общество или новая наука об обществе? Социология мобильностей Джона Урри. М., Праксис, 576 с.

13. Ходыкин А.В. 2019а. Систематизация трактовок взаимосвязи физического и социального пространств в социологических теориях. В кн.: Будущее социологического знания и вызовы социальных трансформаций (к 90-летию со дня рождения В.А. Ядова). М., ФНИСЦ РАН, 285-290.

14. Ходыкин А.В. 2019б. Освоение космоса как социологическая проблема. Социологическое обозрение, 4: 47-73. DOI: 10.17323/1728-192x-2019-4-47-73.

15. Хоружий С.С. 1997. Род или не род? Заметки к онтологии виртуальности. Вопросы философии, 6: 56-74.

16. Lefebvre H. 1974. La production de l'espace. L'Homme et la société, 31(1): 15-32.

17. Urry J. 1999. Automobility, Car Culture and Weightless Travel. Available at: http://www.comp.lancaster.ac.uk/sociology/soc008ju.html (accessed 8 February 2020).

18. Urry J. 2004. The "System" of Automobility. Theory, Culture and Society, 21: 25-39.

19. Urry J. 2000. The Tourist Gaze: Leisure and Travel in Contemporary Societies. London, Sage, $176 \mathrm{p}$.

20. Urry J. 1990. The consumption of tourism. Sociology, 24(1): 23-35.

21. Urry J. 2003. Global Complexity. Cambridge, Polity Press, 172 p.

22. Featherstone M., Thrift N., Urry J. 2005. Automobilities. London, Sage, 285 p.

\section{References}

1. Baudrillard J. 1981. Simulacres et simulation. Paris, Pabl. Galilée, 212 p.

2. Gritsanov A.A., Galkin D.V., Karpenko I.D. 2003. Virtual reality. In: Virtual'naya real'nost' [Virtual reality]. Novejshij filosofskij slovar' / Sost. i gl. nauch. red. A.A. Gricanov. 3-e izd., ispravl. Moscow, Knizhnyj Dom: 171-173.

3. Zvonovsky V.B. 2009. Sociology of everyday life space. In: Sociologiya prostranstva povsednevnosti [Sociology of everyday life space]. Samara, Pabl. Samarskij universitet, 166 p.

4. Ivanov D.V. 2002. Virtualization of society. Version 2.0. In: Virtualizaciya obshchestva. Versiya 2.0. [Virtualization of society. Version 2.0]. SPb., Pabl. Peterburgskoe Vostokovedenie, 217 p.

5. Ruzavin G.I. 2010. Virtuality In Virtual'nost' [Virtuality]. Available at: https://iphlib.ru/library/collection/newphilenc/document/HASH01b7eff9048db8416a128fb7 (accessed 8 February 2020) (in Russian)

6. Sorokin P. 1992. An Individual. Civilization. Society. Moscow, Pabl. Politizdat.

7. Urry J. 2007. Mobilities. London, Pabl. Polity, 335 p.

8. Urry J. 2014. Offshoring. Cambridge, Pabl. Polity, 200 p.

9. Filippov A.F. 2008. Sociology of space. In: Sociologiya prostranstva [Sociology of space]. Saint Petersburg, Pabl. Vladimir Dal', 285 p. 
10. Filippov A.F. 2001. Sociology of space as a theoretical alternative. In: Sociologiya prostranstva kak teoreticheskaya al'ternativa [Sociology of space as a theoretical alternative]. Rossiya transformiruyushcheesya obshchestvo. Moscow, KANON-press-C: 45-63.

11. Filippov A.F. 2003. Theoretical foundations of the sociology of space. In: Teoreticheskie osnovaniya sociologii prostranstva [Theoretical foundations of the sociology of space]. Abstract.. dis. ... dokt. soc. sciences. Moscow, $40 \mathrm{p}$.

12. Kharlamov N.A. 2012. A new society or a new science of society? In: Novoe obshchestvo ili novaya nauka ob obshchestve? [A new society or a new science of society?]. Mobil'nosti. Moscow, Pabl. Praksis, $576 \mathrm{p}$.

13. Khodykin A.V. 2019a. Systematization of interpretations of the relationship between physical and social spaces in sociological theories. In: Sistematizaciya traktovok vzaimosvyazi fizicheskogo i social'nogo prostranstv v sociologicheskih teoriyah [Systematization of interpretations of the relationship between physical and social spaces in sociological theories]. Budushchee sociologicheskogo znaniya i vyzovy social'nyh transformacij (k 90-letiyu so dnya rozhdeniya V.A. Yadova). Moscow, Pabl. FNISC RAN: 285-290.

14. Khodykin A.V. 2019 b. Outer Space Exploration as a sociological problem. In: Osvoenie kosmosa kak sociologicheskaya problema [Outer Space Exploration as a sociological problem]. Sociologicheskoe obozrenie, 4: 47-73. DOI: 10.17323/1728-192x-2019-4-47-73.

15. Horuzhiy S.S. 1997. Genus or not genus? Notes on the ontology of virtuality. In: Rod ili ne rod? Zametki k ontologii virtual'nosti [Genus or not genus? Notes on the ontology of virtuality]. Voprosy filosofii, 6: 56-74.

16. Lefebvre H. 1974. La production de l'espace. L'Homme et la société, 31(1): 15-32.

17. Urry J. 1999. Automobility, Car Culture and Weightless Travel. Available at: http://www.comp.lancaster. ac.uk/sociology/soc008ju.html (accessed 8 February 2020).

18. Urry J. 2004. The "System" of Automobility. Theory, Culture and Society, 21: 25-39.

19. Urry J. 2000. The Tourist Gaze: Leisure and Travel in Contemporary Societies, 2nd edn. Sage, London, $176 \mathrm{p}$.

20. Urry J. 1990. The consumption of tourism. Sociology, 24(1): 23-35.

21. Urry J. 2003. Global Complexity. Cambridge, Polity Press, 172 p.

22. Featherstone M., Thrift N., Urry J. 2005. Automobilities. London, Sage, 285 p.

\section{ИНФОРМАЦИЯ ОБ АВТОРЕ}

Ходыкин Александр Владимирович, аспирант кафедры социологии и психологии института менеджмента Самарского государственного экономического университета, г. Самара, Россия

\section{INFORMATION ABOUT THE AUTHOR}

Alexander V. Khodykin, PhD-student of the Department of sociology and psychology, Institute of management, Samara State Economic University, Samara, Russia 